\title{
Immediate effects of neuromuscular control exercise on neck pain, range of motion, and proprioception in persons with neck pain
}

\author{
Jae-Doo Lee ${ }^{a(1)}$, Won-Seob Shin ${ }^{a, b(1)}$ \\ ${ }^{a}$ Department of Physical Therapy, Graduate School of Health and Medicine, Daejeon University, Republic of Korea \\ ${ }^{\mathrm{b}}$ Department of Physical Therapy, College of Health and Medical Science, Daejeon University, Republic of Korea
}

Objective: This study aimed to investigate the immediate effects of intervention using neuromuscular control, self-stretching (SS), and neck stabilization exercises (NSEs) on neck pain, range of motion (ROM), and proprioception of position sense in adults with neck discomfort.

Design: Three-group pretest-posttest design.

Methods: Forty-four adults who complained of neck pain participated in the experiment. They were randomly assigned to the following groups: neuromuscular control exercise (NMCE) group $(n=15)$, SS group $(n=14)$ and NSE group ( $n=15)$. The NMCE group did rolling with only upper limb pattern on both sides. The SS group performed neck stretching on each side, 3 sets of 30 seconds for each muscle. The NSE group had the pressure biofeedback applied with increases in pressure by $2 \mathrm{mmHg}$ at a time from 20-30 mmHg while in the hook-lying position. All groups performed exercises for 10 minutes. Neck pain, ROM, and proprioception were measured to determine differences between the intervention methods.

Results: Intra-group comparisons showed significant improvement after exercise in pain, ROM, and proprioception in the NMC group $(p<0.05)$. In the comparison between groups, the NMC group had a significant decrease in pain compared to the other two groups $(p<0.05)$. There was no difference in ROM between the groups but the NMC group showed significant improvement in left rotation compared to the stabilization exercise group $(p<0.05)$. For proprioception, the NMC group had significantly lower error than the other two groups $(p<0.05)$.

Conclusions: NMCEs through upper extremity pattern rolling exercise is effective in improving neck pain, ROM, and proprioception.

Key Words: Exercise, Movenet, Neck pain, Proprioception, Range of motion

\section{Introduction}

Although census studies show that approximately onethird of adults experience neck pain or stiffness at least once a week [1], neck related diseases increased by $16.6 \%$ in 2015 compared to 2011 according to data released by the Korea Health Insurance Review \& Assessment Service in 2016 [2]. The main symptoms of persons with neck pain include pain [3], decreased range of motion (ROM) [4], muscle weakness
[5], dysfunction [6], and errors in joint position sense [7], and also differences in electromyography results of the neck muscles from that of the general public [8]. Most individuals with neck pain have impaired proprioception and a decrease in postural stability, thus confusing the afferent information transmitted from the neck [9]. Neck pain and muscle fatigue affect the intrinsic proprioception of the neck [7,10] and posture also affects the function of the proprioceptive sense of the neck [11]. Stretching the musles around the neck and

Received: 14 November, 2019 Revised: 2 December, 2019 Accepted: 9 December, 2019

Corresponding author: Won-Seob Shin (ORCID https://orcid.org/0000-0002-6515-7020)

Department of Physical Therapy, College of Health and Medical Science, Daejeon University, 62 Daehak-ro, Dong-gu, Daejeon 34520, Republic of Korea Tel: 82-42-280-2294 Fax: 82-42-280-2295 E-mail: shinws@dju.kr

(c) This is an Open-Access article distributed under the terms of the Creative Commons Attribution Non-Commercial License (http://creativecommons.org/licenses/ by-nc/4.0) which permits unrestricted non-commercial use, distribution, and reproduction in any medium, provided the original work is properly cited.

Copyright (๑) 2020 Korean Academy of Physical Therapy Rehabilitation Science 
performing neck stabilization exercises (NSEs) are commonly used in the clinic as a method of solving cervical problems. Stretching stimulates the proprioceptive receptors by stretching the muscles and tendons, preventing muscle deterioration, removing fatigue substances, and improving elasticity of the entire muscle tissue $[12,13]$. Several previous studies have suggested that NSEs improve neck pain and deep muscle activity $[14,15]$. Stabilization is the ability of a person to consciously or unconsciously control large or fine movements of a joint [16]. Stabilization exercise had significant effects on pain and psychological improvement in individuals with chronic neck pain [17,18]. Stabilization is not only required for the neck, but it is also required for the nerve roots of the body, strength and endurance, and flexibility [19].

In addition, it is reported that approximately $80 \%$ of neck problems are caused by weakness and reduced flexibility of the muscles around the neck and shoulders [20].

Neck problems require a comprehensive assessment of not only the neck but also of the torso and upper limb movements. As a way of improving the integrated movements of the neck and shoulders, upper limb pattern rolling exercise can be performed in order to improve the neuromuscular control of the head, neck, and core muscles [21]. Previous studies have reported that there was a significant difference in the muscle activation time of the muscles around the hip according to the availability of upper limb pattern rolling exercise, and improvement of preliminary contraction of the stabilizing muscle through the performance of the upper limb pattern rolling exercise has been shown [22]. However, previous studies on the effect of neuromuscular control exercise (NMCE) of the upper limb pattern on the function of the neck are still insufficient.

Therefore, in this study, in order to find out whether NMCE using upper limb pattern rolling exercise can affect not only the hip but also the neck, this study was conducted to investigate whether there is a significant difference in neck pain, ROM, and proprioception of individuals with complaints of neck discomfort. In this study, if the effect of NMCE using the upper limb pattern rolling exercise is found to be effective compared to stretching and stabilization exercise for those who complain of neck pain, decreased ROM, and impaired proprioception, it can be suggested as a useful method to solving proprioception issues.

\section{Methods}

\section{Participants}

Forty-four healthy men and women between twenty and fifty years of age volunteered to participate in the study through recruitment advertisements. The age distribution of each group was homogeneously distributed by age group and the contents are shown in Table 1. The conditions for selecting subjects were those who scored from 5 to less than 15 points on the Neck Disability Index, those who experienced neck pain lasting more than 3 months within the past year, those with neck discomfort within 7 days before this study, and those with a positive Passive Scapular Elevation Test.

Exclusion conditions were those with upper body rotation of less than 50 degrees in a seated posture, pain during upper limb pattern rolling exercises, cervical fracture, arthritis, tumor, muscle disease, osteoporosis, severe neck instability, radiation pain due to compression of nerve tissue, and psychiatric issues that may cause difficulty in comprehending the procedures of the study. Those who understood the purpose of this study agreed to participate in writing. The $\mathrm{G}^{*}$ power Software (version 3.1; Heinrich-Heine-Universität, Düsseldorf, Germany) was used to calculate the effect size by calculating the value of the main variables in the groups before and after intervention as according to a study by Joo

Table 1. General characteristics of subjects

\begin{tabular}{lcccc}
\hline \multicolumn{1}{c}{ Variable } & NMCE $(\mathrm{n}=15)$ & Stretching $(\mathrm{n}=14)$ & Stability $(\mathrm{n}=15)$ & $\chi^{2} / \mathrm{F}(p)$ \\
\hline Sex (male/female) & $8 / 7$ & $6 / 8$ & $6 / 9$ & $0.594(0.371)$ \\
Age $(\mathrm{y})$ & $32.60(7.49)$ & $33.43(9.15)$ & $34.20(9.64)$ & $45.554(0.245)$ \\
$20-30$ & 6 & 6 & 6 & \\
$30-40$ & 6 & 3 & 6 & \\
$40-50$ & 3 & $167.21(8.29)$ & $168.33(8.17)$ & $37.697(0.330)$ \\
Height $(\mathrm{cm})$ & $166.87(8.25)$ & $65.14(13.63)$ & $62.07(12.05)$ & $41.765(0.325)$ \\
Weight $(\mathrm{kg})$ & $66.33(12.18)$ & & & \\
\hline
\end{tabular}

NMCE, neuromuscular control exercise.

Values are presented as number only or mean (SD). 
[23]. After calculating the effect size, the minimum number of target subjects was checked for 42 , and proceeded to 44 in consideration of the dropout rate. This research was conducted under the review of the Institutional Bioethics Committee of Daejeon University (IRB No. 1040647-201910HR-004).

\section{Intervention}

Subjects complaining of neck discomfort were randomly drawn from the numbers on the Neck Disability Index test during the selection process with 15 subjects assigned to the NMCE group, 14 subjects assigned to the self-stretching (SS) group, and 15 subjects assigned to the NSE group. All three groups were assessed for neck pain levels, ROM, and proprioception. The interventions were performed for 10 minutes in each group. After intervention, all three groups had undergone reevaluation of neck pain levels, ROM, and proprioception.

In order to reduce the level of error, all interventions and evaluations were conducted by a physiotherapist who had more than 5 years of musculoskeletal therapy experience.

\section{Neuromuscular control exercise}

Neuromuscular control was mediated by upper limb pattern rolling exercises.

According to the intervention method proposed by Barbara et al. [21], the rolling exercises were performed in the supine-to-prone and prone-to-supine positions.

The rotational force of the neck and arm was used to be able to roll sideways without the use of the lower body as much as possible. In order to limit the use of the lower body, when any force has entered into the lower body or active movements appeared just before when the upper body rota- tion alone forced the body's center to move to the opposite side of the body, then the exercise was stopped and retraining was performed. Torsion of the body through rotational movements of the upper body was emphasized, and the subject was instructed to fully feel the transfer of the force being transmitted from the neck movement to the body. With inclusion of the rest period, the test was conducted for $10 \mathrm{mi}-$ nutes, with 5 minutes each for left and right (Figure 1).

\section{Self-stretch}

SS of the upper trapezius, levator scapula, and the sternocleidomastoid muscles were performed in sitting position [24]. The upper trapezius muscle stretch was performed by using one hand to pull the side of the head in the opposite direction and the opposite arm extended downwards so that the subject could sense the muscle becoming elongated.

The levator scapula was stretched by having one hand grasping the opposite side of the head and pulling the chin towards the direction of the armpit. At the same time, the opposite arm was lifted up along the wall so that the shoulder blade could be turned upward in order to elongate the muscle as much as possible.

To stretch the sternocleidomastoid muscle, the subject was instructed to look back behind their shoulder and lift their head up toward the ceiling while the opposite hand was placed on the forehead and pulled in the direction to elongate the muscle.

All three sets of stretches for the left and right muscles were performed for 30 seconds, with a 30 -second rest period between each set, for approximately 10 minutes (Figure 2).

\section{Neck stabilization exercise}

Pressure biofeedback equipment was applied during the

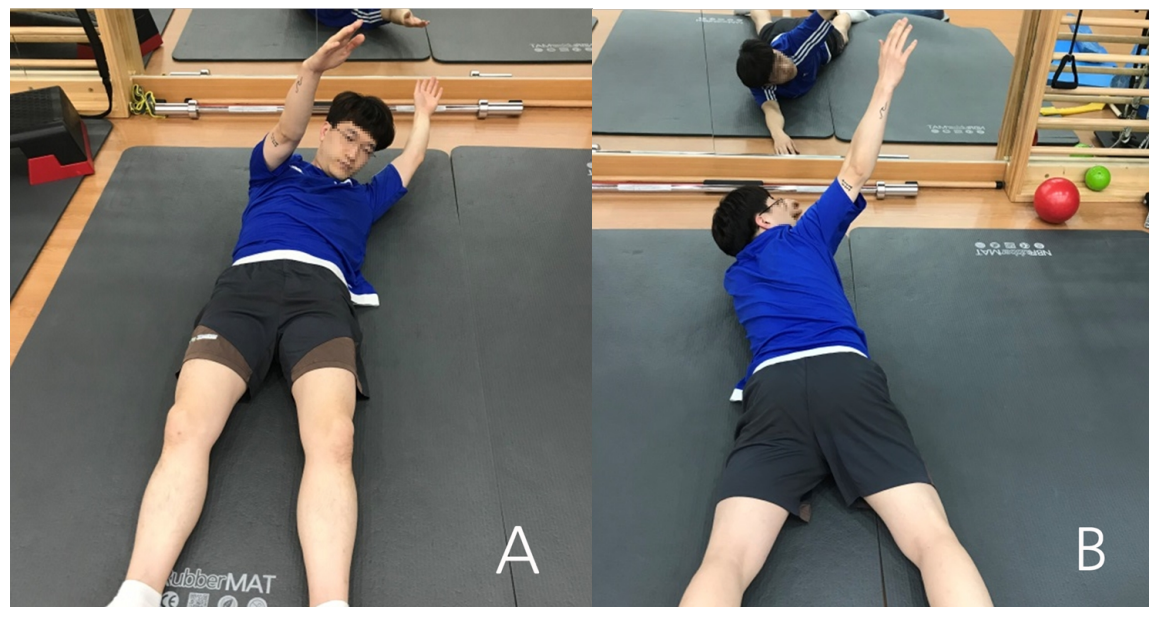

Figure 1. Neuromuscular control exercise. (A) Supine to prone with upper body rolling. (B) Prone to supine with upper body rolling. 


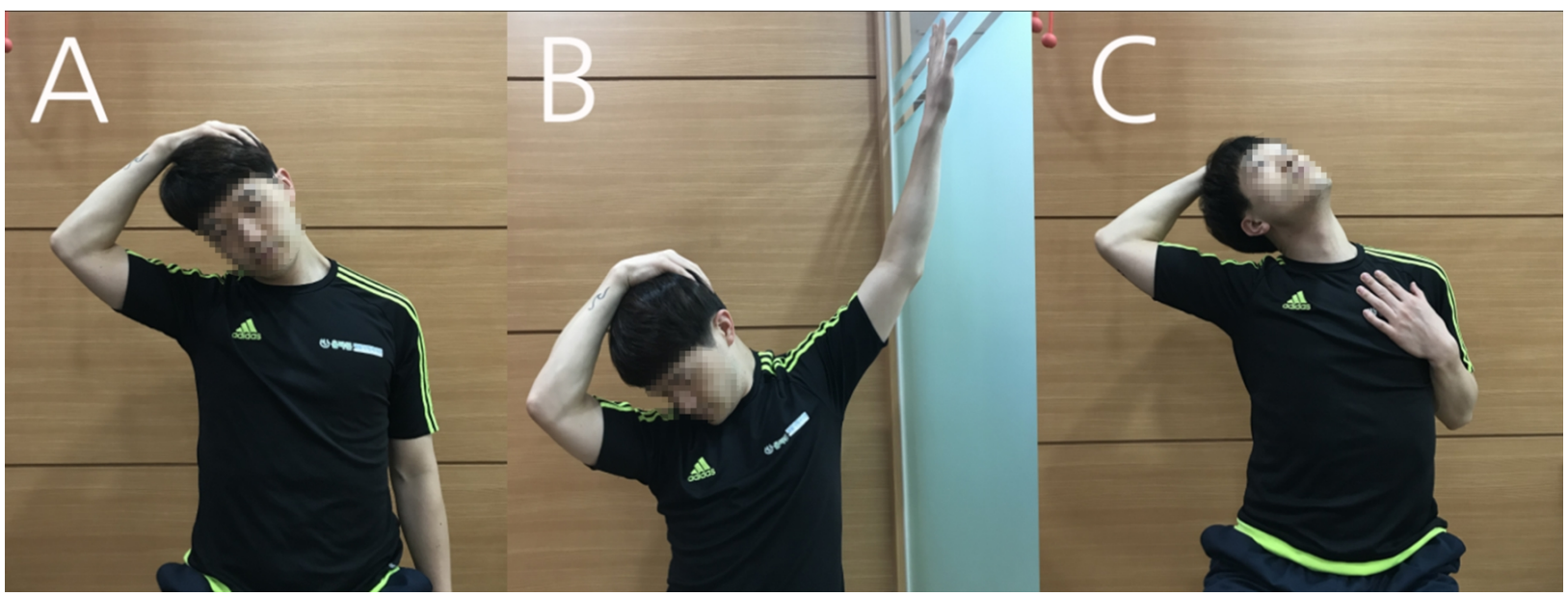

Figure 2. Self-Stretching. (A) Upper trapezius. (B) Levator scapulae. (C) Sternocleidomastoid.

performance of NSEs. With the subject in supine position and with bilateral knees bent, a pressure biofeedback was placed under the neck and was set at $20 \mathrm{mmHg}$ of pressure. The pressure was gradually increased from $20 \mathrm{mmHg}$ to 30 $\mathrm{mmHg}$ by $2 \mathrm{mmHg}$ at a time. The pressure was maintained for 10 seconds for every $2 \mathrm{mmHg}$ increment. The pressure biofeedback was applied for approximately 10 minutes with the inclusion of a 30 -second rest period placed between each set [25].

\section{Outcome measures}

\section{Neck pain}

Pain levels were assessed using the Visual Analogue Scale (VAS). On an unmarked score card, the subjects indicated the extent of pain they were currently experiencing. The degree of pain was assessed by measuring the distance from the starting point to the marked point. The subjects' level of pain was marked on a $100 \mathrm{~mm}$ ruler where the far left end was defined as a comfortable state without pain while the far right end was defined as the most extreme level of pain. The reliability of this assessment is Intraclass Correlation Coefficients $(\mathrm{ICCs})=0.76^{-0.84}$ [26].

\section{Range of motion}

Cervical joint ROM was performed and assessed in cervical flexion, extension, right and left rotation as according to previous studies. All subjects sat on the same chair for the assessment of cervical flexion, and all subject were in supine position on a mat when assessing for cervical rotation. The subjects wore bands on their heads so that they could apply their smartphones on their head while assuming a supine position.

The application of the smartphone and goniometer was located on the left side of the ear canal in between the position of neck flexion and extension with the stationary arm in line with the tongue depressor that was securely fastened in the subject's mouth, and the moving arm placed perpendicular to the stationary arm.

For cervical rotation, the axis was placed at the top center of the head, the stationary arm was parallel to the ground or parallel to the acromion process, and the moving arm was in line with the nose. Cervical joint rotational ROM was measured starting from the anatomical position to full range.

An inclinometer-based measurement application for smartphones (Iphone 7; Apple Inc., Cupertino, CA, USA) was used in this study, which has an ICC range from 0.72 to 0.94 [27].

\section{Proprioception (position sense)}

The Head Repositioning Accuracy (HRA) was used to assess neck proprioception, which assesses head repositioning [28]. Subjects were seated in a backless chair with the back straightened and hands placed comfortably on the thigh. The subjects wore a band with a laser point (Motion Guidance, West Valley City, UT, USA) on their head and the distance between the subject and the target was adjusted to $90 \mathrm{~cm}$.

The target was made from a $60 \mathrm{~mm} \times 80 \mathrm{~cm}$ piece of graph paper based on a $1 \mathrm{~mm}$ scale and the intersection of horiztonal and vertical lines was drawn. In order to set the zero point for the along the horizontal plane for the HRA measurement, after performing maximal cervical extension with eyes closed, when the subject faced the front, the head was 
adjusted by moving the center of the intersection line to the location of the laser point. Next, once the subjects were allowed to align the laser to a new center point with their eyes opened, they were instructed to close their eyes while maintaining this position for 5 seconds in order to remember the new location. After the subjects were instructed to perform maximal left cervical rotation and then return to facing the front, the distance between the intersection and the laser point was measured. This procedure was performed 10 times and the average was obtained. The same procedure was performed for right cervical rotation as well. The reliability of this assessment is 0.43 to 0.91 [29] (Figure 3).

\section{Data and statistical analysis}

Data was collected and analyzed by IBM SPSS Statistics for Windows, Version 25.0 (IBM Co., Armonk, NY, USA). The general characteristics of the subjects were presented as descriptive statistics and mean and standard deviation values. The homogeneity was confirmed by the Chi-square test and One-way ANOVA. For the normality test of all variables the Shapiro-Wilks test was performed. For comparisons of preand post intervention results within the group the paired t-test was conducted and analyzed. Post-hoc analysis was performed using the Bonferroni correction. The statistical significance of this study was set to $\alpha=0.05$.

\section{Results}

A total of 44 subjects participated in this study, including

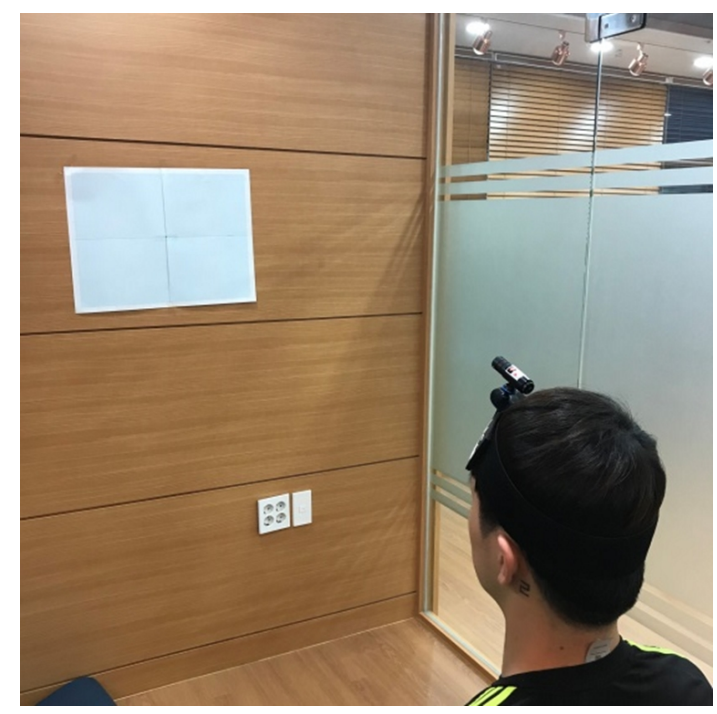

Figure 3. Proprioception of position sense. the NMCE group (n1=15), the SS group (n2=14), and the stabilization exercise group $(\mathrm{n} 3=15)$. The general characteristics of the subjects are shown in Table 1. Pain test results showed significant effects in the pre- and post-group comparisons in the NMCE group, the SS group, and the stabilization exercise group $(p<0.01)$. In comparison of the preand post-changes between the three groups, NMCEs were observed to be more effective compared to the SS and stabilization exercises $(p<0.01)$.

For cervical joint ROM, significant pre- and post-intervention effects were observed between the neuromuscular control group, SS group, and stabilization exercise group. There were significant effects in the NMCE group and the SS group in pre- and post intervention comparisons. In the pre- and post-neck comparisons of right neck rotation, the NMCE group, the SS group, and the stabilization exercise group had significant effects. There was a significant effect in the pre- and post-neck comparisons of left cervical rotation in the neuromuscular control group and the SS group $(p<0.01)$. In comparison of the pre and post changes between the three groups, the NMCE was more effective than the stabilization exercise group for left cervical rotation range $(p<0.05)$.

Based on the position sense examination to assess for proprioception, there were significant within-group pre and post intervention effects in the NMCE group for right cervical rotation proprioception $(p<0.01)$.

Intra-neck rotational intrinsic sensation had significant effects in the NMCE group $(p<0.01)$, SS group $(p<0.05)$, and the stabilization exercise group $(p<0.01)$. Based on the comparisons of pre- and post-group changes, neuromuscular control was more effective than SS and stabilization exercise for right and left cervical proprioception $(p<0.01)$ (Table 2).

\section{Discussion}

Neck pain is reported to occur more than once in a lifetime in approximately $67 \%$ of the total population [30], and is more common in office workers and those who work with computers and smartphones over an extended period of time [31].

Neck pain has a high probability of recurrence and has been reported to have a poor prognosis [32]. Among subjects with neck discomfort, this study was conducted to compare the effects of performing the upper extremity pattern rolling exercise, in which this exercise is known to improve 
Table 2. Comparison of pain, ROM, proprioception $(\mathrm{N}=44)$

\begin{tabular}{|c|c|c|c|c|}
\hline Variable & $\operatorname{NMCE}(n=15)$ & Stretching $(n=14)$ & Stability $(\mathrm{n}=15)$ & $\mathrm{F}$ \\
\hline \multicolumn{5}{|l|}{ VAS (cm) } \\
\hline Pre & $4.33(0.80)$ & $4.54(0.89)$ & $4.01(0.76)$ & 1.575 \\
\hline Post & $2.87(0.87)$ & $4.07(1.02)$ & $3.35(1.10)$ & $5.287 * *$ \\
\hline $\mathrm{t}(p)$ & $6.924(<0.001)$ & $2.900(0.006)$ & $3.413(0.002)$ & \\
\hline Change & $-1.46(0.82)^{\mathrm{a}, \mathrm{b}}$ & $-0.47(0.61)$ & $-0.65(0.74)$ & $7.649 * *$ \\
\hline \multicolumn{5}{|l|}{ Flexion $\left({ }^{\circ}\right)$} \\
\hline Pre & $54.27(11.49)$ & $53.36(10.37)$ & $59.87(11.2)$ & 1.492 \\
\hline Post & $60.00(9.21)$ & $55.57(10.32)$ & $63.87(7.66)$ & 3.012 \\
\hline $\mathrm{t}(p)$ & $-3.913(0.001)$ & $-3.910(0.001)$ & $-2.770(0.007)$ & \\
\hline Change & $5.73(5.66)$ & $2.21(2.12)$ & $4.00(5.59)$ & 1.941 \\
\hline \multicolumn{5}{|l|}{ Extension $\left({ }^{\circ}\right)$} \\
\hline Pre & $68.00(7.90)$ & $66.29(7.25)$ & $64.33(6.76)$ & 0.946 \\
\hline Post & $71.13(7.20)$ & $68.79(6.30)$ & $64.33(6.76)$ & $4.113^{*}$ \\
\hline $\mathrm{t}(p)$ & $-1.870(0.041)$ & $-4.167(<0.001)$ & $0.000(0.500)$ & \\
\hline Change & $3.13(6.91)$ & $2.50(2.24)$ & $0.00(6.81)$ & 1.334 \\
\hline \multicolumn{5}{|l|}{ Right rotation $\left(^{\circ}\right)$} \\
\hline Pre & $66.87(7.71)$ & $67.00(3.01)$ & $69.53(7.89)$ & 0.754 \\
\hline Post & $72.33(4.25)$ & $69.93(2.87)$ & $72.87(7.46)$ & 1.263 \\
\hline $\mathrm{t}(p)$ & $-3.410(0.002)$ & $-7.614(<0.001)$ & $-5.054(<0.001)$ & \\
\hline Change & $5.47(6.21)$ & $2.93(1.44)$ & $3.33(2.550)$ & 1.707 \\
\hline \multicolumn{5}{|l|}{ Left rotation $\left(^{\circ}\right)$} \\
\hline Pre & $65.53(5.95)$ & $66.14(5.36)$ & $71.27(7.75)$ & 3.538 \\
\hline Post & $73.67(4.78)$ & 70.57 (5.18) & $73.20(6.19)$ & 1.358 \\
\hline $\mathrm{t}(p)$ & $-5.150(<0.001)$ & $-4.855(<0.001)$ & $-1.112(0.142)$ & \\
\hline Change & $8.13(6.17)^{\mathrm{b}}$ & $4.43(3.41)$ & $1.93(6.73)$ & $4.566^{*}$ \\
\hline \multicolumn{5}{|c|}{ Proprioceptive right rotation $(\mathrm{cm})$} \\
\hline Pre & $24.43(9.38)$ & $25.60(9.17)$ & $24.69(9.08)$ & 0.064 \\
\hline Post & $16.67(6.28)$ & $25.64(8.43)$ & $23.08(8.84)$ & $5.384 * *$ \\
\hline $\mathrm{t}(p)$ & $3.701(0.001)$ & $-0.143(0.444)$ & $1.054(0.155)$ & \\
\hline Change & $-7.76(8.12)^{a, b}$ & $0.32(8.60)$ & $-0.95(3.48)$ & $5.562 * *$ \\
\hline \multicolumn{5}{|c|}{ Proprioceptive left rotation $(\mathrm{cm})$} \\
\hline Pre & $23.88(9.19)$ & $23.04(6.24)$ & $24.00(10.06)$ & 0.051 \\
\hline Post & $15.27(5.36)$ & $20.43(7.36)$ & $20.84(8.59)$ & 3.166 \\
\hline $\mathrm{t}(p)$ & $5.349(<0.001)$ & $2.132(0.026)$ & $1.641(0.061)$ & \\
\hline Change & $-8.65(6.26)^{\mathrm{a}, \mathrm{b}}$ & $-1.90(3.33)$ & $-3.16(7.46)$ & $5.281 * *$ \\
\hline
\end{tabular}

Values are presented as mean (SD).

ROM: range of motion, NMCE: neuromuscular control exercise, VAS: Visual Analogue Scale.

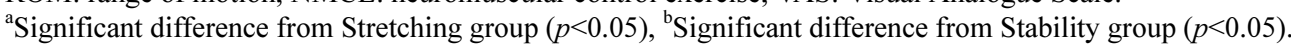

${ }^{*} p<0.05,{ }^{* *} p<0.01$.

spinal stability and head-neck coordination ability, through the NMCE group, and to compare with the effects of SS, and NSE on neck pain, joint ROM, and proprioception.

There were significant effect on VAS scores in all three groups in the pre- and post-group comparisons $(p<0.01)$. This result was in part consistent with the finding that Hwang et al. [33] where they reported significantly reduced pain in the SS group in a previous study that mediated SS and upper spine arthroplasty in 37 patients over 8 weeks. Evjenth and Hamberg [24] suggested that stretching is the most fun- damental and effective method for treating joint contractions or pain. Joo [23] reported a significant reduction in pain after 4 weeks of NSEs with use of pressure biofeedback. In comparison of neck pain between groups before and after intervention, NMCE was found to be more effective than the SS group and stabilization exercise group $(p<0.01)$.

This results suggest that NMCEs are more effective than SS and NSEs.

With regards to intervention of the cervical area, an integrative view of the muscles and nerves should be more em- 
phasized, and it is related to previous studies that sensorymotor training, which is an integrated program of musclebone-joint and neurological control systems, is effective in controlling posture and maintaining stability [19].

In regards to cervical joint range, pre-post intervention comparison of the cervical flexion angle showed that there were significant effects in all three groups.

There were significant effects on the cervical extension angle in the NMCE group and SS group in the pre- and postinternal comparisons, but there was no significant difference in the stabilization exercise group.

There was a significant effect in all three groups in the pre- and post intervention comparisons of the right cervical rotation angle.

There was a significant effect in the NMCE group and the SS group in the pre- and post intervention comparisons of the left cervical rotation angle $(p<0.01)$. Jeon et al. [34] found that 4 weeks of performing stretch exercises significantly improved neck flexion, extension, and left-right rotation ROM in elderly patients. In this study, performing self-stretches also showed significant improvement in cervical joint flexion, extension, and left-right rotation range.

In comparison of the pre- and post-variable changes among the three groups, the NMCE group had a greater significant effect on left cervical movement range compared to the stabilization exercise group $(p<0.05)$. There was no significant difference in comparison between neuromuscular exercise group and SS group.

In a previous study, subjects with neck pain were classified according to whether they've tested positive or negative on the Passive Scapular Elevation Test. After being on 4 weeks of a shoulder stabilization exercise program, the study had found that there were significant differences in pain levels, dysfunction levels, and neck ROM in those who've tested positive compared to those who've tested negative on the Passive Scapular Elevation Test [35].

In this study, subjects were selected by the manual shoulder bone raising test [36], which is used to screen for mechanical problems of the neck and shoulder muscles. It can be seen that more dynamic changes can be made to the muscles around the back [36]. Based on the position sense examination performed to assess for proprioception, accordin gto the within-group comparisions, there were significant effects on right cervical rotation propropception in the NMCE group $(p<0.01)$. There were significant effects on intra-neck rotational intrinsic proprioception in the NMCE group $(p<0.01)$, SS group $(p<0.05)$ and stabilization exercise group $(p<0.01)$.
Jull et al. [20] compared the effects of cranio-cervical flexion and proprioceptive training for 6 weeks in subjects with chronic neck pain. In both cases, cevical joint position sense was significantly decreased. In another study, subjects with chronic neck pain were found to have fewer joint position sense errors with four weeks of cervcial proprioception rehabilitation compared to conventional cervical rehabilitation [37].

In contrast, no significant difference was found between cranial injury patients and normal subjects when they measured cranio-cervical flexion and immediately measured joint position sense [38]. The experimental results were partially in agreement with the results of no significant difference in neck stabilization through cranial neck bending. As a result of comparing the pre and post change between groups, the neuromuscular control group had more significant effects than the SS and the stabilization exercise group $(p<0.01)$.

As a result, the effect of NMCE is more effective than self stretching and stabilization exercise on the improvement of proprioceptive sensation.

Previous studies on neck problems have been conducted in various ways and the effectiveness of exercise has been demonstrated. However, the approach to neuromuscular control of the neck has not been robustly studied compared to the intervention of other body parts. Based on the results of this study, various exercise therapy approaches to the neck need to be attempted.

The limitation of this study was that the immediate effect was first tested and therefore, the duration of the intervention is cannot be known precisely. Second, since the experiment was conducted on subjects with mild neck discomfort, it is not known how it may affect individuals with moderate to severe pain and limitations.

If further research is conducted that can change the duration of intervention and provide more change to the subjects, a variety of exercise therapy interventions may become available for individuals with neck pain.

\section{Conflict of Interest}

The authors declared no potential conflicts of interest with respect to the authorship and/or publication of this article.

\section{References}

1. Gordon SJ, Trott P, Grimmer KA. Waking cervical pain and stiff- 
ness, headache, scapular or arm pain: gender and age effects. Aust J Phys 2002;48:9-15.

2. Health Insurance Review \& Assessment Service. 2016 National health insurance statistical yearbook. Seoul: Health Insurance Review \& Assessment Service; 2017.

3. Jette DU, Jette AM. Physical therapy and health outcomes in patients with spinal impairments. Phys Ther 1997;76:930-41; discussion 942-5.

4. Olson SL, O'Connor DP, Birmingham G, Broman P, Herrera L. Tender point sensitivity, range of motion, and perceived disability in subjects with neck pain. J Orthop Sports Phys Ther 2000;30:13-20.

5. Silverman JL, Rodriquez AA, Agre JC. Quantitative cervical flexor strength in healthy subjects and in subjects with mechanical neck pain. Arch Phys Med Rehabil 1991;72:679-81.

6. Jull G, Barrett C, Magee R, Ho P. Further clinical clarification of the muscle dysfunction in cervical headache. Cephalalgia 1999; 19:179-85.

7. Chen X, Treleaven J. The effect of neck torsion on joint position error in subjects with chronic neck pain. Man Ther 2013;18:5627.

8. Kim GE, Yun DU, An YJ, Park DS, Ham JH. Reliability and validity of new evaluation methods using static surface electromyography in persons with neck pain. Phys Ther Rehab Sci 2019; $8: 1-7$.

9. Cheng CH, Wang JL, Lin JJ, Wang SF, Lin KH. Position accuracy and electromyographic responses during head reposition in young adults with chronic neck pain. J Electro Kines 2010;20: 1014-20.

10. Reddy RS, Maiya AG, Rao SK. Effect of dorsal neck muscle fatigue on cervicocephalic kinaesthetic sensibility. Hong Kong Phys J 2012;30:105-9.

11. Jung JM, Gu JS, Shin WS. The effect of different sitting postures on range of motion, strength and proprioceptive sense of neck. J Korea Acad Ind Coop Soc 2012;13:2212-8.

12. Lee CJ. The effects of recovery trials of stretching on physiological fatigue variables during high-intensity weight training [Master thesis]. Daegu: Keimyung University; 2006.

13. Jeong HM, Shim JH, Hye RS. The passive stretching, massage, and muscle energy technique effects on range of motion, strength, and pressure pain threshold in musculoskeletal neck pain of young adults. Phys Ther Rehabil Sci 2017;6:196-201.

14. Childs JD, Cleland JA, Elliott JM, Teyhen DS, Wainner RS, Whitman JM, et al. Neck pain: clinical practice guidelines linked to the international classification of functioning, disability, and health from the orthopedic section of the American Physical Therapy Association. J Orthop Sports Phys Ther 2008;38:A1A34.

15. Ferreira PH, Ferreira ML, Maher CG, Herbert RD, Refshauge K. Specific stabilisation exercise for spinal and pelvic pain: a systematic review. Aust J Physiother 2006;52:79-88.

16. Lee S, Park J, Lee D. Effects of bridge exercise performed on an unstable surface on lumbar stabilizing muscles according to the knee angle. J Phys Ther Sci 2015;27:2633-5.

17. Kim GC, HwangBo PN. Effects of cervical stabilization exercise using pressure biofeedback on neck pain, forward head posture and acoustic characteristics of chronic neck pain patients with forward head posture. J Korean Soc Phys Med 2019;14:121-9.
18. Seo SC, Choi JY, Joo MY, Kim JH, Chang SK. Effects of sling exercise and McKenzie exercise program on neck disability, pain, muscle strength and range of motion in chronic neck pain. Phys Ther Rehabil Sci 2012;1:40-8.

19. Kwon JH, Cho MJ, Park MC, Kim SY. Cervical stabilization exercise using the Sling system. J Korean Acad Orthop Man Ther 2002;8:57-71.

20. Jull GA, Falla D, Vicenzino B, Hodges PW. The effect of therapeutic exercise on activation of the deep cervical flexor muscles in people with chronic neck pain. Man Ther 2009;14:696-701.

21. Hoogenboom BJ, Voight ML, Cook G, Gill L. Using rolling to develop neuromuscular control and coordination of the core and extremities of athletes. N Am J Sports Phys Ther 2009;4:70-82.

22. Clark N, Voight ML, Campbell AM, Pierce S, Sells P, Cook R, et al. The relationship between segmental rolling ability and lumbar multifidus activation time. Int J Sports Phys Ther 2017;12: 921-30.

23. Joo MK. The effects of stabilization exercise on chronic neck pain patients [Master thesis]. Daejeon: Daejeon University; 2010.

24. Evjenth O, Hamberg J. Autostretching: the complete manual of specific stretching. Sweden: Alfta Rehab Forlag; 2001.

25. Jull GA. Deep cervical flexor muscle dysfunction in whiplash. J Musculoskelet Pain 2000;8:143-54.

26. Boonstra AM, Schiphorst Preuper HR, Reneman MF, Posthumus JB, Stewart RE. Reliability and validity of the visual analogue scale for disability in patients with chronic musculoskeletal pain. Int J Rehabil Res 2008;31:165-9.

27. Lee C, Song H, Lee J, Chang M. Usefulness of smart phone application to measure cervical range of motion. J Korean Soc Community Based Occup Ther 2017;7:17-24.

28. Rix GD, Bagust J. Cervicocephalic kinesthetic sensibility in patients with chronic, nontraumatic cervical spine pain. Arch Phys Med Rehabil 2001;82:911-9.

29. Wibault J, Vaillant J, Vuillerme N, Dedering Å, Peolsson A. Using the cervical range of motion (CROM) device to assess head repositioning accuracy in individuals with cervical radiculopathy in comparison to neck- healthy individuals. Man Ther 2013;18:403-9.

30. Wang WT, Olson SL, Campbell AH, Hanten WP, Gleeson PB. Effectiveness of physical therapy for patients with neck pain: an individualized approach using a clinical decision-making algorithm. Am J Phys Med Rehabil 2003;82:203-18; quiz 219-21.

31. Jun DH. A prospective investigation into the effects of workplace stress and working postures on work-related neck pain in office workers. PNF Mov 2019;17:253-61.

32. Kjellman G. Neck pain: analysis of prognostic factors and treatment effects [Master thesis]. Linköping: Linköping University; 2001.

33. Hwang BG, Kim GC, Park YK. Effects of self-stretching exercise and upper thoracic joint mobilization on range of motion and pain of the patients with chronic neck pain. J Korean Soc Phys Med 2012;7:509-14.

34. Jeon HY, Lee SY, Bae SS, Jang C, Lee GC. Effect of stretching exercise on cervical ROM in elderly. J Korean Soc Phys Med 2009;4:57-62.

35. Kim HY, Kim SY, Jang HJ, Joo MK. Effect of scapular stabilization exercise on patients with neck pain classified according to passive scapular elevation test. Phys Ther Korea 2012;19:51-60. 
36. McDonnell MK, Sahrmann SA, Van Dillen L. A specific exercise program and modification of postural alignment for treatment of cervicogenic headache: a case report. J Orthop Sports Phys Ther 2005;35:3-15.

37. Ravi CN, Dibyendunarayan BD, Ramalingam TA. Effectiveness of proprioceptive exercises in chronic nonspecific neck pain patients. Indian J Phys Occup Ther 2016;10:143-8.

38. Armstrong BS, McNair PJ, Williams M. Head and neck position sense in whiplash patients and healthy individuals and the effect of the cranio-cervical flexion action. Clin Biomech (Bristol, Avon) 2005;20:675-84. 